ARTICLE

Received 3 Mar 2015 | Accepted 8 Oct 2015 | Published 3 Nov 2015

DOI: $10.1038 /$ ncomms 9826

OPEN

\title{
Onset of two-dimensional superconductivity in space charge doped few-layer molybdenum disulfide
}

\author{
Johan Biscaras ${ }^{1}$, Zhesheng Chen ${ }^{1}$, Andrea Paradisi ${ }^{1} \&$ Abhay Shukla ${ }^{1}$
}

\begin{abstract}
Atomically thin films of layered materials such as molybdenum disulfide $\left(\mathrm{MoS}_{2}\right)$ are of growing interest for the study of phase transitions in two-dimensions through electrostatic doping. Electrostatic doping techniques giving access to high carrier densities are needed to achieve such phase transitions. Here we develop a method of electrostatic doping which allows us to reach a maximum $n$-doping density of $4 \times 10^{14} \mathrm{~cm}^{-2}$ in few-layer $\mathrm{MoS}_{2}$ on glass substrates. With increasing carrier density we first induce an insulator to metal transition and subsequently an incomplete metal to superconductor transition in $\mathrm{MoS}_{2}$ with critical temperature $\approx 10 \mathrm{~K}$. Contrary to earlier reports, after the onset of superconductivity, the superconducting transition temperature does not depend on the carrier density. Our doping method and the results we obtain in $\mathrm{MoS}_{2}$ for samples as thin as bilayers indicates the potential of this approach.
\end{abstract}

\footnotetext{
${ }^{1}$ Institut de Minéralogie, de Physique des Matériaux et de Cosmochimie (IMPMC), Sorbonne Universités-UPMC Univ. Paris 06, UMR CNRS 7590, Muséum National d'Histoire Naturelle, IRD UMR 206, 4 Place Jussieu, 75005 Paris, France. Correspondence and requests for materials should be addressed to A.S. (email: abhay.shukla@impmc.upmc.fr).
} 
ayered materials are being extensively studied for their exceptional properties when reduced to few atomic layers. The promise of producing innovative electronic devices is alluring. So is the perspective of electrostatic doping to induce substantial carrier density and phase transitions ${ }^{1}$ in two-dimensions (2D). Recent research in both fields has widely used molybdenum disulphide $\left(\mathrm{MoS}_{2}\right)$ as a prototype.

Bulk $\mathrm{MoS}_{2}$ is a semiconductor with an indirect electronic band-gap. In the limit of the single layer a direct gap semiconductor is obtained ${ }^{2,3}$ as the indirect transition energy becomes larger than the direct gap at the $K, K^{\prime}$ points of the Brillouin Zone. The strong light-matter interaction at these points ${ }^{4}$ and the lifting of the spin degeneracy of the valence and conduction bands due to inversion symmetry breaking make single layer $\mathrm{MoS}_{2}$ an excellent material for transistors in electronics, opto-electronics and the developing valleytronics field ${ }^{5-8}$.

Chemical intercalation can induce an $n$-doped metallic and superconducting state ${ }^{9,10}$, a fact that has inspired electrostatic and electrochemical doping of few-layer $\mathrm{MoS}_{2}$. The insulator-metal transition and carrier densities of about $10^{13} \mathrm{~cm}^{-2}$ have been reported using solid dielectrics like $\mathrm{SiO}_{2}$ (refs 11,12) and $\mathrm{HfO}_{2}$ (ref. 13). Using an ionic liquid as a dielectric, densities above $10^{14} \mathrm{~cm}^{-2}$ could be obtained with superconductivity appearing in a narrow region between $8 \times 10^{13}$ and $3 \times 10^{14} \mathrm{~cm}^{-2}$ and a maximum critical temperature of $11 \mathrm{~K}$ (refs 14,15). Both these techniques involve delicate device issues and whereas a solid dielectric does not allow for very high doping, the liquid gating method requires great care to avoid possible intercalation, electrochemical reactions ${ }^{16}$ and strain during the liquid to solid transformation of the dielectric, which may be avoided by using a solid ionic conductor ${ }^{17}$.

Here we develop a method of electrostatic doping that allows us to reach a maximum $n$-doping density of $4 \times 10^{14} \mathrm{~cm}^{-2}$ in the simplest of devices consisting of few-layer $\mathrm{MoS}_{2}$ on glass. We produce few-layer $\mathrm{MoS}_{2}$ samples with our anodic bonding method $^{18-20}$ that uses a glass substrate containing mobile ionic species at high temperatures ( $\approx 400 \mathrm{~K}$ or higher). A layered sample is placed on the substrate and an electric field induces an ionic drift and a space charge at the sample-substrate interface leading to a very strong electrostatic field. The electric field 'sticks' the layered sample to the glass substrate and mechanical exfoliation of the attached sample leaves few-layer residue on the glass substrate. Our 'space charge doping' technique ${ }^{21}$ is a natural extension of this method for which we start with the fewlayer sample lying on the glass substrate (either soda-lime glass or borosilicate glass). At high temperature, the glass substrate can be seen as a negatively charged static matrix (mainly $\mathrm{SiO}_{2}$ units) with mobile positively charged ionic species depending on the glass composition, in our case $\mathrm{Na}^{+}$ions ${ }^{22}$. Applying a positive (resp. negative) voltage on the gate electrode at the back of the substrate generates a drift current of mobile $\mathrm{Na}^{+}$going towards (resp. away from) the sample and creating an accumulation (resp. depletion) layer below the interface as shown in Fig. 1. This space charge at the interface induces electrostatic doping of the fewlayer sample. Mobility of $\mathrm{Na}^{+}$in glass in our experimental conditions remains small, which means that charging can be finely controlled and measured in real time over minutes to hours. Thus, by applying a positive or negative gate voltage of the order of $1-100 \mathrm{~V}$, the sample can be doped ambivalently with a consequent variation of its resistivity, which can be monitored along with the gate current as a function of time. The doping process can be quenched at a particular value by cooling the sample to room temperature or below, thus 'freezing' the $\mathrm{Na}^{+}$ ions in the glass matrix and annulling the ionic mobility and drift current. The carrier density that depends on the total charge transferred during the doping process is determined in this static state by Hall effect measurements. Heating of the polarized substrate causes a drift current which tends to nullify the space charge layer and thus remove electrostatic doping of the sample. This process can be accelerated by the application of an appropriate electric field. The technique allows to dope/un-dope the samples multiple times with consistent results, even after ex-situ storage. Raman spectra of samples before and after doping cycles do not show any signs of modification (Supplementary Fig. 1 and Supplementary Note 1) though returning to an undoped state after very high doping $\left(>10^{14} \mathrm{~cm}^{-2}\right)$ can sometimes result in an increase in residual resistivity possibly due to mechanical strain in the sample. In this study, we show the result of space charge doping applied to few-layer $\mathrm{MoS}_{2}$ with varying thickness. An insulator-metal transition is induced at moderate doping. The very high carrier density accessible with our doping technique allows us to subsequently induce superconductivity.
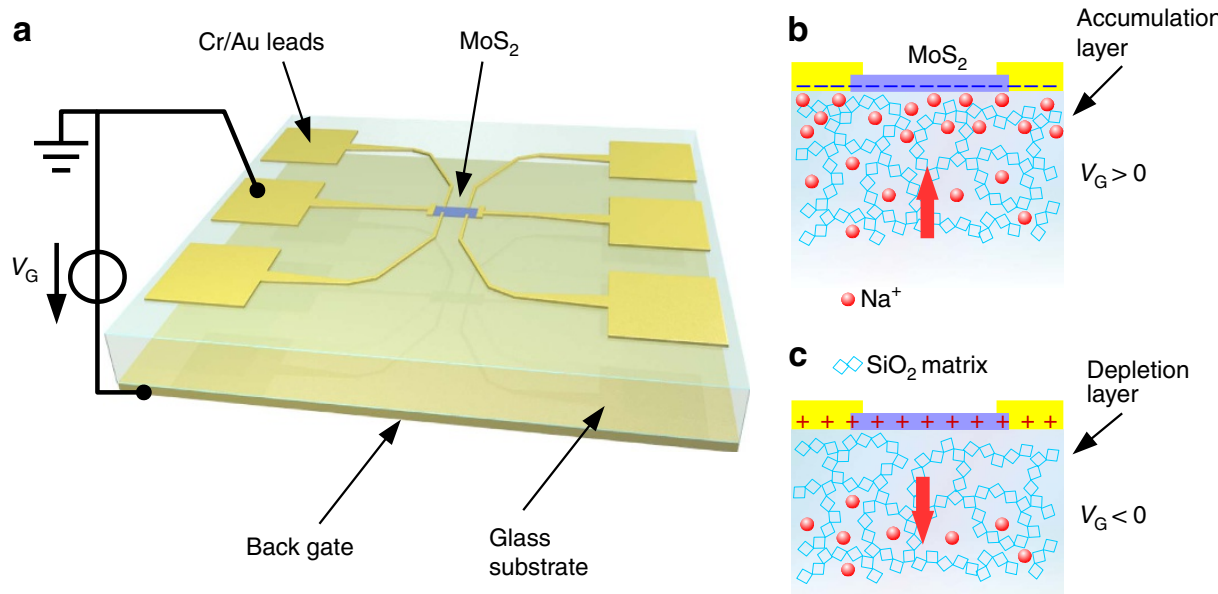

Figure 1 | Space charge doping configuration. (a) Schematic 3D view of the space charge doping set-up with few-layer MoS $\mathrm{S}_{2}$ contacted in a Hall bar pattern on a back-gated glass substrate. $(\mathbf{b}, \mathbf{c})$, Schematic view of the cross section of the $\mathrm{MoS}_{2}$ /glass substrate interface showing the creation of an accumulation (resp. depletion) layer by the movement of sodium ions under the applied electric field (red arrow). 


\section{Results}

Sample characterization. $\mathrm{MoS}_{2}$ samples are prepared and contacted as described in the Methods section. As prepared samples typically show resistance of the order of G $\Omega$ in ambient conditions. Transport measurements are performed in a high vacuum cryostat coupled to an external 2-T electromagnet for Hall measurements. After annealing in the cryostat at $420 \mathrm{~K}$ for several hours the resistance decreases to $20-100 \mathrm{k} \Omega$. The properties of $\mathrm{MoS}_{2}$ have been shown to be particularly sensitive to adsorbates, especially in the few-layer limit ${ }^{23,24}$, explaining this observation. The annealing also ensures the removal of any residual space charge from the sample fabrication step. The initial $n$-doping density is found to be around $10^{13} \mathrm{~cm}^{-2}$. This substantial initial doping originates from shallow $\mathrm{Na}^{+}$donor impurity levels due to interfacial ions ${ }^{25}$ as we show below.

Insulator-Metal transition. We show results from three samples with varying thickness: $2 \mathrm{~nm}$ (bilayer according to Raman measurements), $4.5 \mathrm{~nm}(\sim 7$ layer $)$ and $11 \mathrm{~nm}$ ( $\sim 17$ layer $)$. An example of the space charge doping procedure is shown in Fig. 2. This procedure involves doping the sample at high temperature $(\approx 400 \mathrm{~K})$ by application of a gate voltage during a time determined by the desired change in resistivity, quenching to room temperature followed by resistivity and Hall measurements as a function of temperature down to $\sim 3 \mathrm{~K}$. The insulator-metal transition occurring in the low to moderate carrier density range $\left(0.3-5 \times 10^{13} \mathrm{~cm}^{-2}\right)$ is clearly visible for all samples, as shown in Fig. 3. As the doping dynamics is slow and electrostatic equilibrium is never reached in our experiments (Fig. 2), the absolute value of the gate voltage used during the polarization is not relevant. Indeed, the same carrier density can be obtained with a lower gate voltage but longer time, as what counts is the accumulated space charge. The metallic state is reached by applying a positive gate voltage ( $n$-doping), the insulator-metal transition (indicated by a horizontal dashed line in the figure) always occurring around the quantum of resistance $\left(R_{\mathrm{Q}}=h / e^{2}\right)$ despite differences in mobility and number of layers, consistent with previous reports ${ }^{15}$. This transition corresponds to the Mott-Ioffe-Regel limit ${ }^{26}$ in $2 \mathrm{D}$ when the electronic wavelength is of the same order of magnitude as the mean free path, that is $k_{\mathrm{F}} l \sim 1$.

It is worth noting that low temperature localization behaviour $(\partial R / \partial T<0$, refs 27,28$)$ can be seen on the metallic side of the metal-insulator transition. In the inset of Fig. $3 c$ we show an example of such localization for the $2 \mathrm{~nm}$ sample at a doping of $2 \times 10^{13} \mathrm{~cm}^{-2}$ and at $4.7 \mathrm{~K}$ through the measurement of negative magnetoresistance. This behaviour originates from quantum interference corrections to the conductivity (electrons are backscattered coherently after multiple collisions) and thus is only seen at low temperature, while the metal-insulator transition corresponding to the quantum of resistance originates from strong localization of electronic wave-functions (the mean free path is shorter than the electronic wavelength and the electrons do not propagate). It is thus seen at much higher temperatures $(300 \mathrm{~K})$.

The transition can be reversed by hole doping (with a negative gate voltage) which pushes the sample back into the insulating state. Low temperature $I-V$ characteristics in the insulating state show non-Ohmic behaviour typical of variable range hopping (Supplementary Figs 2 and 3 and Supplementary Note 2).

The carrier densities are measured by Hall effect. All the measured Hall resistances $R_{\mathrm{H}}(B)$ show linear behaviour with the magnetic field in the range we used (Fig. 4a) which allows to determine the Hall carrier density through the formula $R_{\mathrm{H}} / B=1 / e n_{\mathrm{S}}$. The carrier concentration decreases with temperature as shown in Fig. 4c-e. The cation mobility in the glass being negligible below $300 \mathrm{~K}$, this effect is not due to a loss of polarization in the glass and has been verified to depend only on temperature and not on applied gate voltage. This can be attributed to the formation of shallow donor impurity levels in the gap near the bottom of the conduction bands by the $\mathrm{Na}^{+}$
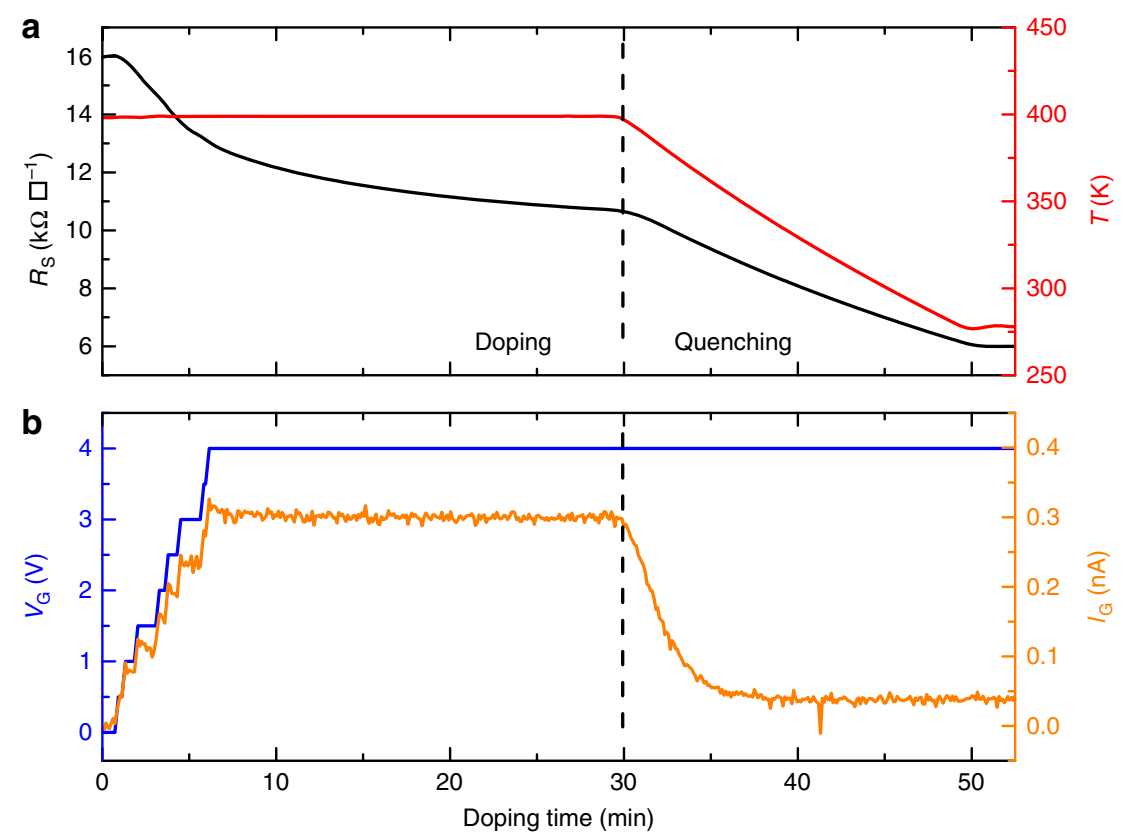

Figure 2 I Space charge doping dynamics. (a) Sheet resistivity (black line, left axis) of a 2-nm thick sample as a function of doping time during space charge doping experiment with positive gate voltage at $T=400 \mathrm{~K}$ and the subsequent quenching in temperature. The temperature is shown as the red curve (right axis), the vertical dash line marks the limit between the doping itself and the following temperature quench. (b) Applied gate voltage (blue line, left axis) and gate current measured (orange line, right axis) during the space charge doping experiment. 

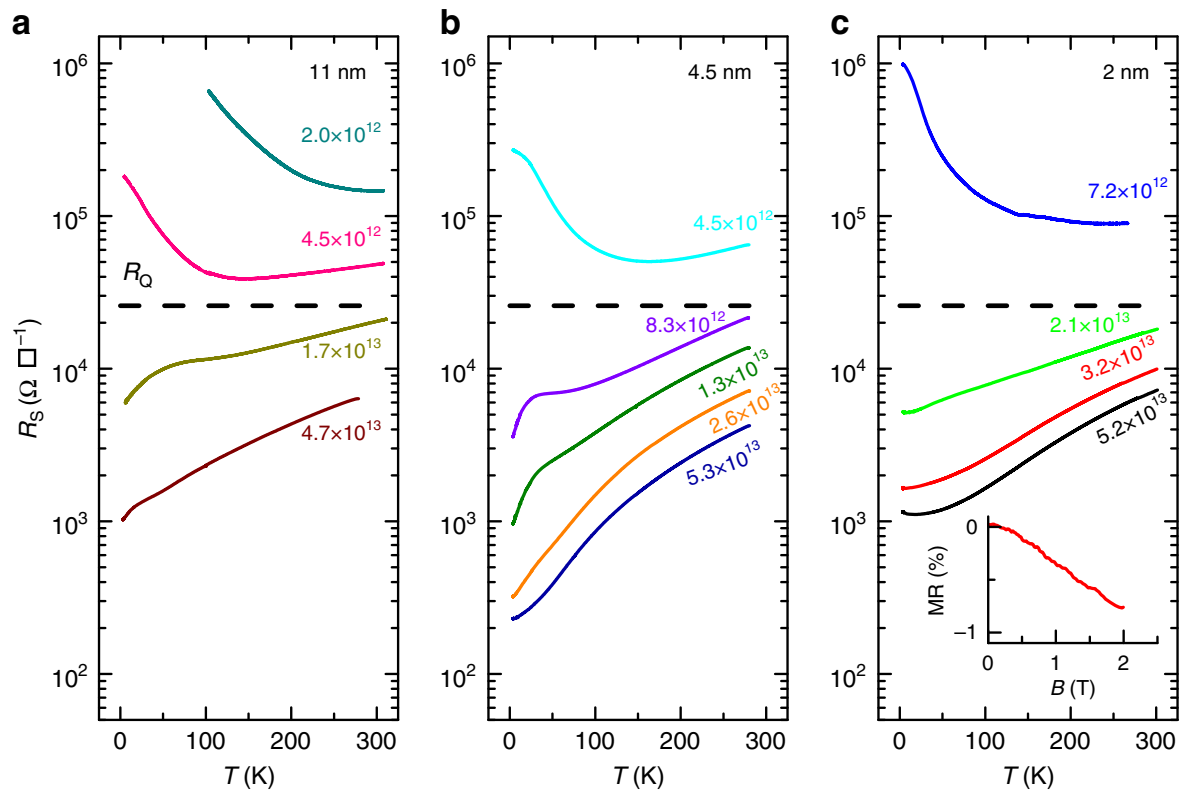

Figure 3 | Metal-Insulator transition. (a-c), Sheet resistance as a function of temperature of the three $\mathrm{MoS}_{2}$ samples for low to moderate doping levels achieved with space charge doping. The carrier densities measured at room temperature are displayed next to each curve (in units of $\mathrm{cm}^{-2}$ ). The samples $(11,4.5$ and $2 \mathrm{~nm})$ are labelled by thickness as measured by AFM. The horizontal dashed line is the quantum of resistance $R_{\mathrm{Q}}=h / e^{2}$. The inset of panel $\mathbf{c}$ shows the magnetoresistance $M R=\Delta R / R$ of the $2 \mathrm{~nm}$ sample as a function of perpendicular magnetic field at $4.7 \mathrm{~K}$ during the doping experiment corresponding to the red curve of the main panel.

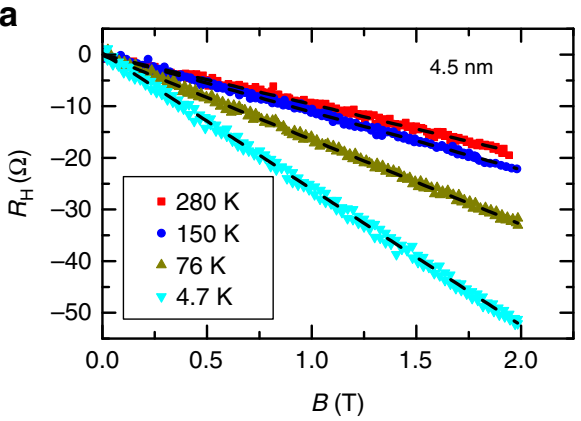

C

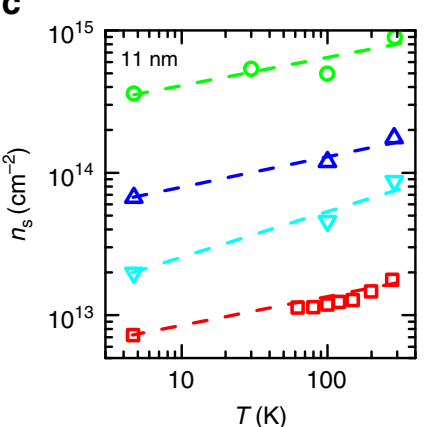

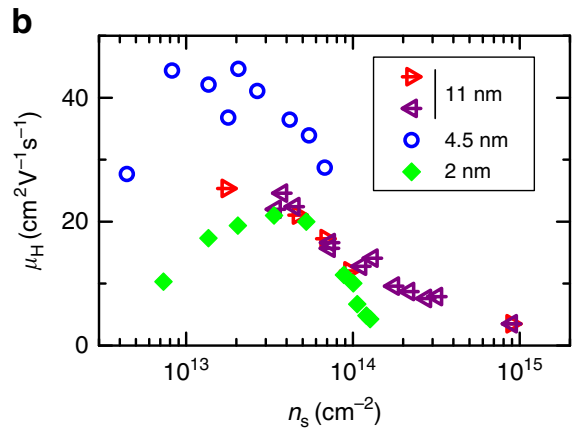

e

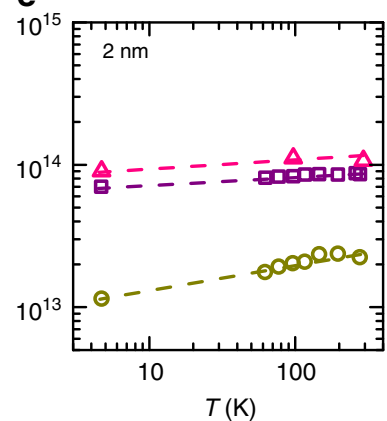

Figure 4 | Hall effect. (a) Anti-symmetrized Hall resistance as a function of magnetic field of the $4.5 \mathrm{~nm}$ sample measured at a space charge induced carrier density of $\sim 2$ to $5 \times 10^{13} \mathrm{~cm}^{-2}$ at different temperatures, showing a decrease in carrier density at low temperatures due to impurity level trapping. Black dashed lines are linear fits. (b) Hall mobility of the three samples measured at $280 \mathrm{~K}$ as a function of the space charge induced carrier density showing an increase with doping at low density and decrease at high carrier density. For the $11 \mathrm{~nm}$ sample the red arrows pointing to the right are measurements made while increasing the carrier density, while the purple arrows pointing to the left are made while decreasing carrier density after maximum doping. (c,d,e), Carrier density variation with temperature at some fixed doping levels for each sample. Dashed lines are guides for the eye.

ions at the glass surface ${ }^{25}$. These traps can capture electrons from the $\mathrm{MoS}_{2}$ sample at low temperature, thus reducing the carrier density, while at high temperatures they are ionized resulting in more efficient space charge doping. Carrier densities are extracted directly from the Hall coefficient assuming a single band with isotropic mass and diffusion coefficient. High doping could 
involve carriers in multiple valleys with different band mass because of the complex structure of the $\mathrm{MoS}_{2}$ conduction band ${ }^{15}$. However, recent ab-initio band calculations ${ }^{29}$ show that for electrostatically $n$-doped $\mathrm{MoS}_{2}$ the one band Hall constant formula holds even beyond carrier densities of $10^{14} \mathrm{~cm}^{-2}$, with small variations $(20 \%)$ at $4 \times 10^{14} \mathrm{~cm}^{-2}$. A simple model assuming two bands implies non-linear Hall resistance behaviour not seen in our measurements.

The mobilities derived from the Hall carrier density at high temperature $(280 \mathrm{~K})$ are shown in Fig. 4 b. At low carrier density, the mobility increases with carrier density as it has been reported in solid states FET devices ${ }^{12}$, whereas the high density mobility is limited by increased scattering processes ${ }^{30-32}$. There is also the possibility that the electrostatic pressure at very high doping may locally deform the crystal lattice to adapt to the roughness of the amorphous glass substrate. Indeed at densities $\sim 10^{14} \mathrm{~cm}^{-2}$ we can estimate that the electrostatic pressure reaches $e^{2} n_{\mathrm{S}}^{2} / \epsilon_{0} \sim$ $3 \mathrm{GPa}$ that is significantly smaller but not negligible compared to the Young's modulus of $\sim 0.3 \mathrm{TPa}$ and breaking stress of $\sim 20 \mathrm{GPa}$ of monolayer $\mathrm{MoS}_{2}$ (ref. 33). However, $\mathrm{MoS}_{2}$ nanosheets can be deformed reversibly to several nanometres parallel to $c$ axis $^{34}$, while the roughness of our substrates has been measured to be between 0.2 and $0.8 \mathrm{~nm}$ through atomic force microscopy (AFM). Thus the possibility of irreversible deformation is small. Accordingly, mobility before and after maximum doping for the $11 \mathrm{~nm}$ sample does not change as shown in Fig. 4 b, however, irreversible deformation in the $2 \mathrm{~nm}$ sample may be possible at very high doping.

At low temperatures $(4.7 \mathrm{~K})$ the mobilities expectedly increase enormously due to reduced phonon scattering. The $2 \mathrm{~nm}$ sample showed mobilities from 80 to $200 \mathrm{~cm}^{2} \mathrm{~V}^{-1} \mathrm{~s}^{-1}$ for low to moderate $\left(\sim 10^{13} \mathrm{~cm}^{-2}\right)$ doping. The thicker $11 \mathrm{~nm}$ sample had mobilities from 200 to $700 \mathrm{~cm}^{2} \mathrm{~V}^{-1} \mathrm{~s}^{-1}$ in the same doping range. The intermediate $4.5 \mathrm{~nm}$ sample displayed high mobilities up to $1,800 \mathrm{~cm}^{2} \mathrm{~V}^{-1} \mathrm{~s}^{-1}$ at low doping, decreasing to $\sim 1,300 \mathrm{~cm}^{2} \mathrm{~V}^{-1} \mathrm{~s}^{-1}$ at $2 \times 10^{13} \mathrm{~cm}^{-2}$. These values are in agreement with previous reports on $\mathrm{MoS}_{2}$ in these doping regimes ${ }^{35,36}$. The differences in mobilities in the three samples are most likely related to the quality of the initial bulk samples.

Superconductivity. Beyond the insulator to metallic transition which occurs at moderate doping, the samples were doped to higher carrier densities. The maximum carrier densities measured at room temperature were $1.4 \times 10^{14} \mathrm{~cm}^{-2}$ for the $2 \mathrm{~nm}$ sample, $7.8 \times 10^{13} \mathrm{~cm}^{-2}$ for the $4.5 \mathrm{~nm}$ sample and $8.9 \times 10^{14} \mathrm{~cm}^{-2}$ for the $11 \mathrm{~nm}$ sample. The much higher value obtained for the $11 \mathrm{~nm}$ sample is explained by the fact that it is placed on glass with a higher sodium concentration (soda-lime instead of borosilicate glass). At the relatively low maximum carrier density achieved in the $4.5 \mathrm{~nm}$ sample no superconducting transition was found.
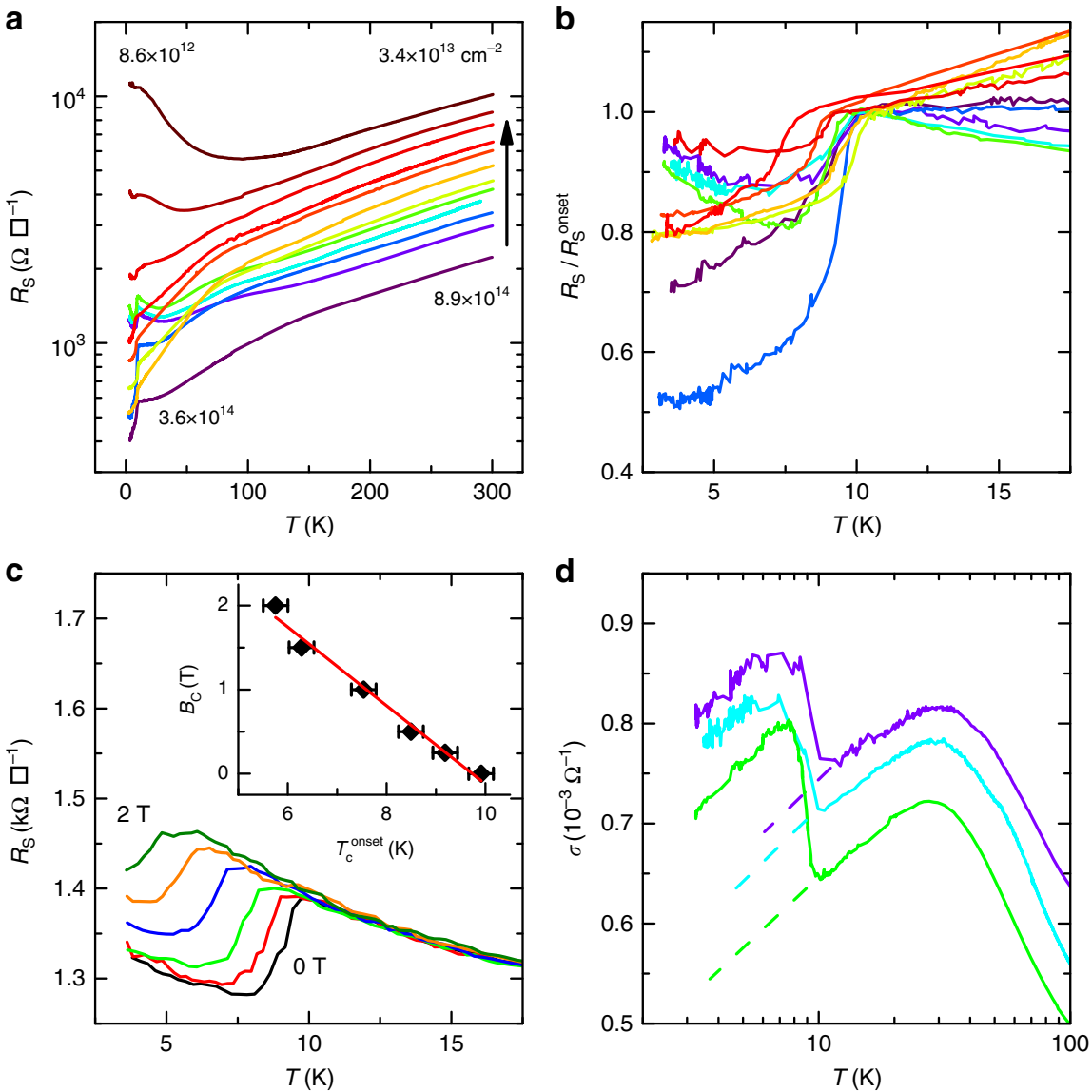

Figure 5 | High doping regime of $\mathbf{1 1} \mathbf{~ n m ~ s a m p l e . ~ ( a ) , ~ S h e e t ~ r e s i s t a n c e ~ o f ~ t h e ~} 11 \mathrm{~nm}$ sample as a function of temperature for high doping levels. The arrow indicates the sequence of measurements. The carrier densities displayed (in units of $\mathrm{cm}^{-2}$ ) are measured by Hall effect at room temperature and $4.7 \mathrm{~K}$ for the two extremal curves, showing the range of measurements. (b), Sheet resistance normalized by its onset value as a function of temperature showing incomplete superconductivity. (c), Sheet resistance as a function of temperature under perpendicular magnetic field $(0,0.25,0.5,1,1.5$ and $2 \mathrm{~T})$ at fixed carrier density $\left(\sim 2 \times 10^{13} \mathrm{~cm}^{-2}\right)$. Inset: Critical field $B_{C}$ as a function of onset critical temperature, with linear fit (red line). The error bars relate to the extraction of the onset critical temperature. (d), Weak localization on both sides of the superconducting transition at some doping densities extracted from panel $\mathbf{b}$ 

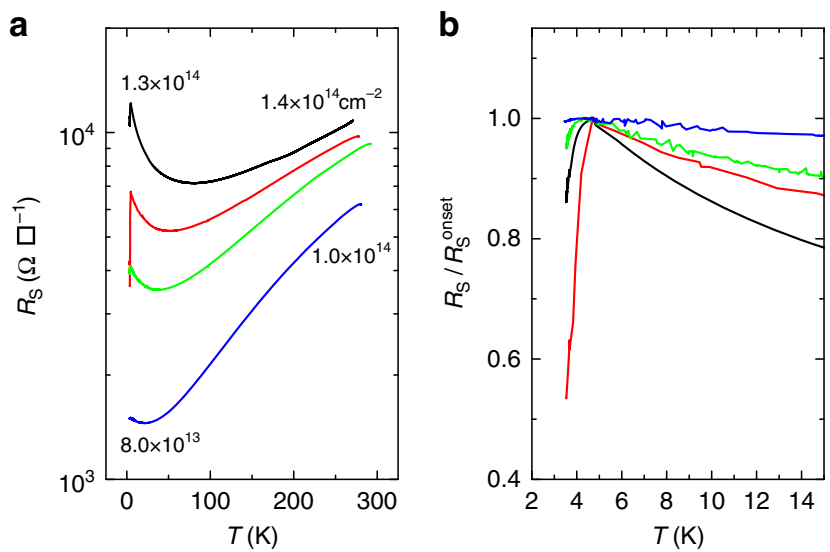

Figure 6 | High doping regime of the $\mathbf{2} \mathbf{~ n m}$ sample. (a), Sheet resistance of the $2 \mathrm{~nm}$ sample as a function of temperature for high doping levels. The carrier densities displayed (in units of $\mathrm{cm}^{-2}$ ) are measured by Hall effect at room temperature and $4.7 \mathrm{~K}$ for the two extremal curves. (b) Sheet resistance normalized by its onset value as a function of temperature showing incomplete superconductivity.

Figure 5 shows the measurements on the $11 \mathrm{~nm}$ sample that was first doped to the maximum carrier density where a sharp fall in resistance at low temperatures, indicating superconductivity, appeared. Doping was then progressively decreased resulting in the expected monotonic increase in the room temperature sheet resistance and eventual disappearance of superconductivity as shown in Fig. 5a for the whole temperature range. The low temperature sheet resistance evolves in a more complicated manner with decreasing doping. Possible weak localization below $20 \mathrm{~K}$ is seen $(\partial R / \partial T<0)$ coexisting with superconductivity though it disappears at still lower doping. Fig. 5b shows resistivity curves normalized to the superconducting onset temperature in the range up to $20 \mathrm{~K}$. The transition temperature is independent of the doping despite the wide range of measured carrier densities (from $\sim 10^{13}$ to $3 \times 10^{14} \mathrm{~cm}^{-2}$ ) and roughly constant at $\approx 10 \mathrm{~K}$. A signature of the superconducting transition is its dependence on the applied perpendicular magnetic field. As shown in Fig. 5c, the transition shifts to lower temperatures with an increasing perpendicular magnetic field. This well known variation of the transition temperature is approximately linear close to $T_{\mathrm{C}}$ as is observed for the onset superconducting temperature in our sample in the inset of Fig. 5c. By fitting our data to the Bardeen-Cooper-Schrieffer (BCS) formula for this variation we estimate the zero Kelvin critical field to be $B_{\mathrm{C}}(0) \sim 3 \mathrm{~T}$.

As shown in Fig. 6 a the $2 \mathrm{~nm}$ bilayer was doped progressively from low to high carrier concentrations which provoked both an increase in resistance and eventually a superconducting transition. The increase in sheet resistance at the highest doping levels is probably caused by irreversible crystalline deformation in this ultra-thin sample as discussed above. Again, below $20 \mathrm{~K}$ signs of localization $(\partial R / \partial T<0)$ coexist with superconductivity. Despite the low $T_{\mathrm{C}} \approx 4 \mathrm{~K}$ in this sample the onset of the transition is clear.

Both samples thus display an incomplete low temperature superconducting transition with significant differences from the one reported in ref. 15.

First, it is incomplete with non-zero resistance below $T_{\mathrm{C}}$ as can be seen in Fig. 5 and similar to reports in refs 14, 16. This implies the existence of a dissipating normal phase at temperatures lower than $T_{\mathrm{C}}$ (defined as the superconducting onset temperature).

Second, each sample shows this behaviour over doping ranges that are different from those reported earlier. The $11 \mathrm{~nm}$ sample shows a doping independent $\sim 10 \mathrm{~K}$ critical temperature over a

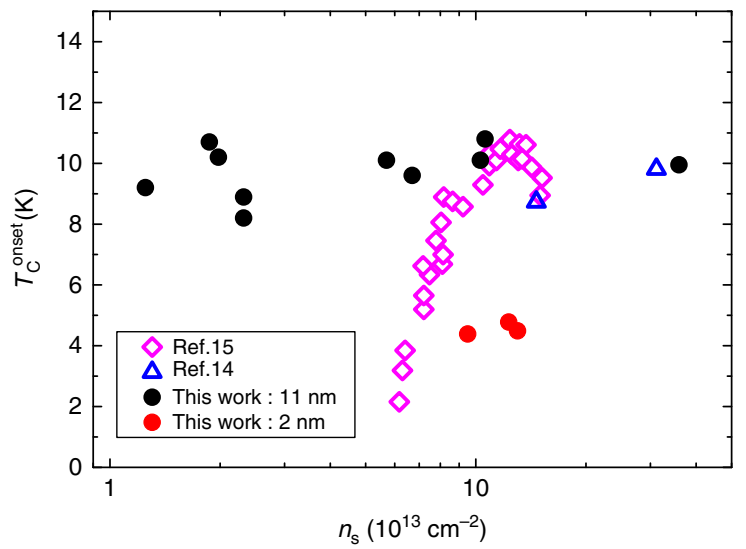

Figure 7 | Superconducting critical temperature. Critical temperature corresponding to the onset of the superconducting transition as a function of Hall carrier density measured at low temperature for the $2 \mathrm{~nm}$ (red circles) and the $11 \mathrm{~nm}$ (black circles) samples. Open symbols represent values reported in ref. 15 (pink open diamonds) and ref. 14 (blue open triangles) and are added for comparison.

large carrier concentration range, from a strikingly low $1.3 \times 10^{13}$ (at which neither $2 \mathrm{~nm}$ nor $4.5 \mathrm{~nm}$ samples are superconducting) to $3.6 \times 10^{14} \mathrm{~cm}^{-2}$ (Fig. 7). The bilayer sample shows a lower $T_{\mathrm{C}} \approx 4 \mathrm{~K}$ from carrier concentrations of $9 \times 10^{13}$ to $1.4 \times 10^{14} \mathrm{~cm}^{-2}$. Though electrostatic doping is probably confined to a thickness corresponding to less than the bilayer in both samples, the higher critical temperature in the thicker sample could be due to a $c$ axis superconducting coherence length in superconducting $\mathrm{MoS}_{2}$ that is greater than the bilayer thickness. Even in the insulating regime the conductivity shows a transition from $2 \mathrm{D}$ to $3 \mathrm{D}$ behaviour in going from the bilayer to the thick sample (Supplementary Note 2).

Third, in some cases superconductivity is preceded by non-metallic behaviour with increase in resistance as temperature decreases $(\partial R / \partial T<0)$, characteristic of localization. As shown in Fig. 5d this localization corresponds to a logarithmic decrease of conductivity with temperature corresponding to $2 \mathrm{D}$ electrical transport ${ }^{27,28}$. Residual fermions continue to localize below $T_{\mathrm{C}}$.

As a cross-check on the reproducibility of our results all three samples were measured at least twice. Between measurements each sample was reduced to its original post-annealing doping state and subsequently removed from the cryostat, measured with Raman spectroscopy and stored in a primary vacuum for days or weeks. Reintroduction into the cryostat followed by doping cycles and measurement gave reproducible results.

\section{Discussion}

Non-zero resistance in superconducting materials is an ongoing matter of research as multiple factors could lead to the appearance of dissipation above and below the superconducting transition, especially in low dimensions. Indeed, thermal vortex generation produces dissipation at any non-zero temperature below $T_{\mathrm{C}}$ in a $2 \mathrm{D}$ superconducting strip ${ }^{37}$, and the finite size of the sample may limit the low temperature divergence of the coherence length of the BKT transition leading to a vanishing resistive tail at low temperature ${ }^{38}$.

On the other hand, non percolating superconducting phases can display saturating non-zero resistance at zero temperature in amorphous systems ${ }^{39}$, or systems with inhomogeneities at low carrier densities ${ }^{40}$. 
As they are produced by exfoliation, our samples are single crystals with low defect density as shown by the measured mobilities. However, the saturation resistivity shown by the $11 \mathrm{~nm}$ sample is too high to be explained by vortices or finite size effects alone. The space charge layer in the amorphous glass substrate may also display inhomogeneities at the nanometre scale. The resulting doping inside the crystalline $\mathrm{MoS}_{2}$, however, should be smoothed out by Coulomb screening, especially at high doping. Finally, the non-zero resistance could be attributed to some other extrinsic factor arising from our unique method. Preliminary measurements on a monolayer high critical temperature superconductor sample show that zero resistance superconductivity is attained in this sample under similar conditions, putting to doubt this last possibility. The saturation resistivity of the $11 \mathrm{~nm}$ sample even at the highest carrier densities is thus intriguing and will require further investigation.

The independence of $T_{\mathrm{C}}$ on carrier density (Fig. 7) is worth noting and different from the results reported in ref. 15 . However, results similar to ours have recently been reported in ionic liquid doped $\mathrm{WS}_{2}$ (ref. 41). BCS superconductivity and the corresponding $T_{\mathrm{C}}$ depend on phonon frequencies, electronphonon coupling and the density of states (DOS) at the Fermi level. In few-layer $\mathrm{MoS}_{2}$, the variations in the DOS with electron doping arise essentially from the particular variation of the conduction band which has two minima, at the $\mathrm{K}$ point and the $\mathrm{Q}$ point in the Brillouin zone ${ }^{29}$. The relative positions of these vary with the number of layers and the DOS changes in a step-like manner when the Fermi level crosses these minima. This could also account for differences in the superconductivity found in the 2 and $11 \mathrm{~nm}$ sample.

In conclusion, we have shown that very high doping densities that are required to trigger superconductivity can be reached by space charge doping using a simple and robust configuration of few-layer samples on glass substrates. This doping is reversible and applicable without risks of sample deterioration or electrochemical contamination which limit and complicate the use of other methods. It is also fully compatible with the anodic bonding method for sample fabrication making it a very competitive and efficient solution for such applications. We illustrate this by measuring the insulator-metal and superconducting transitions in few-layer $\mathrm{MoS}_{2}$. We find incomplete superconductivity independent of doping in a wide carrier density range but sensitive to confinement in ultra-thin samples. These findings provide a means for future studies in a variety of materials.

\section{Methods \\ Sample preparation. Few-layer samples of $\mathrm{MoS}_{2}$ were made by the anodic bonding method ${ }^{18-20}$. Precursor flakes were carefully peeled off from natural molybdenite crystals and deposited on a $0.5 \mathrm{~mm}$ thick glass substrate (either standard soda-lime, or borosilicate Schott D263T). Substrate and precursor were put between two electrodes and heated between 100 and $200{ }^{\circ} \mathrm{C}$ to activate ionic mobility in the glass. Then a high voltage was applied between the two electrodes to attract sodium ions in the glass away from the interface with the precursor. After few minutes, the precursor is electrostatically stuck to the glass and the voltage can be removed. Using adhesive tape, the upper layers of the precursor can be mechanically exfoliated, leaving large area few-layer $\mathrm{MoS}_{2}$ on the glass substrate. The thickness of the samples were evaluated by optical contrast, Raman spectroscopy $^{42}$ and confirmed by AFM topography measurements.}

Measurement details. Electrical contacts were made on selected $\mathrm{MoS}_{2}$ layers in Hall bar geometries by standard electron beam lithography techniques, followed by thermal evaporation of $\mathrm{Cr}(5 \mathrm{~nm})$ and $\mathrm{Au}(70 \mathrm{~nm})$ and liftoff. See Supplementary Fig. 4 for sample pictures. The backside of the glass substrate was then glued by silver epoxy to a gold electrode evaporated on top of an insulating $\mathrm{MgO}$ substrate to act as a back gate. Four point resistivity and Hall measurements were made in a high vacuum $\left(P<10^{-6}\right.$ mbar) Oxford He-flow cryostat with a minimum temperature of $2.8 \mathrm{~K}$ and maximum $420 \mathrm{~K}$. A resistive electromagnet was used to apply magnetic fields up to $2 \mathrm{~T}$ perpendicular to the sample.

\section{References}

1. Ahn, C. H. et al. Electrostatic modification of novel materials. Rev. Mod. Phys. 78, 1185-1212 (2006).

2. Splendiani, A. et al. Emerging photoluminescence in monolayer $\mathrm{MoS}_{2}$. Nano Lett. 10, 1271-1275 (2010).

3. Mak, K. F., Lee, C., Hone, J., Shan, J. \& Heinz, T. F. Atomically thin $\mathrm{MoS}_{2}$ : A new direct-gap semiconductor. Phys. Rev. Lett. 105, 136805 (2010).

4. Mak, K. F. et al. Tightly bound trions in monolayer $\mathrm{MoS}_{2}$. Nat. Mater. 12, 207-211 (2013).

5. Zeng, H., Dai, J., Yao, W., Xiao, D. \& Cui, X. Valley polarization in $\mathrm{MoS}_{2}$ monolayers by optical pumping. Nat. Nanotechnol. 7, 490-493 (2012).

6. Xiao, D., Liu, G.-B., Feng, W., Xu, X. \& Yao, W. Coupled spin and valley physics in monolayers of $\mathrm{MoS}_{2}$ and other group-vi dichalcogenides. Phys. Rev. Lett. 108, 196802 (2012).

7. Sallen, G. et al. Robust optical emission polarization in $\mathrm{MoS}_{2}$ monolayers through selective valley excitation. Phys. Rev. B 86, 081301 (2012).

8. Mak, K. F., He, K., Shan, J. \& Heinz, T. F. Control of valley polarization in monolayer $\mathrm{MoS}_{2}$ by optical helicity. Nat. Nanotechnol. 7, 494-498 (2012).

9. Hermann, A., Somoano, R., Hadek, V. \& Rembaum, A. Electrical resistivity of intercalated molybdenum disulfide. Solid State Commun. 13, 1065-1068 (1973).

10. Woollam, J. A. \& Somoano, R. B. Superconducting critical fields of alkali and alkaline-earth intercalates of $\mathrm{MoS}_{2}$. Phys. Rev. B 13, 3843-3853 (1976).

11. Neal, A. T., Liu, H., Gu, J. \& Ye, P. D. Magneto-transport in $\mathrm{MoS}_{2}$ : Phase coherence, spinorbit scattering, and the hall factor. ACS Nano 7, 7077-7082 (2013).

12. Baugher, B. W. H., Churchill, H. O. H., Yang, Y. \& Jarillo-Herrero, P. Intrinsic electronic transport properties of high-quality monolayer and bilayer $\mathrm{MoS}_{2}$. Nano Lett. 13, 4212-4216 (2013).

13. Radisavljevic, B. \& Kis, A. Mobility engineering and a metal-insulator transition in monolayer $\mathrm{MoS}_{2}$. Nat. Mater. 12, 815-820 (2013).

14. Taniguchi, K., Matsumoto, A., Shimotani, H. \& Takagi, H. Electric-fieldinduced superconductivity at $9.4 \mathrm{~K}$ in a layered transition metal disulphide $\mathrm{MoS}_{2}$. Appl. Phys. Lett. 101, 042603 (2012).

15. Ye, J. T. et al. Superconducting dome in a gate-tuned band insulator. Science 338, 1193-1196 (2012).

16. Ye, J. T. et al. Liquid-gated interface superconductivity on an atomically flat film. Nat. Mater. 9, 125-128 (2010).

17. Taniguchi, K., Fukamichi, T., Itaka, K. \& Takagi, H. Programmable persistent interfacial metallic state induced by frozen ions in inorganicglass solid electrolyte. Adv. Func. Mater. 25, 3043-3048 (2015).

18. Shukla, A., Kumar, R., Mazher, J. \& Balan, A. Graphene made easy: High quality, large-area samples. Solid State Commun. 149, 718-721 (2009).

19. Balan, A. et al. Anodic bonded graphene. J. Phys. D: Appl. Phys 43, 374013 (2010).

20. Gacem, K., Boukhicha, M., Chen, Z. \& Shukla, A. High quality 2D crystals made by anodic bonding: a general technique for layered materials. Nanotechnology 23, 505709 (2012).

21. Paradisi, A., Biscaras, J. \& Shukla, A. Space charge induced electrostatic doping of two-dimensional materials: graphene as a case study. Appl. Phys. Lett. 107, 143103 (2015).

22. Mehrer, H., Imre, A. W. \& Tanguep-Nijokep, E. Diffusion and ionic conduction in oxide glasses. JPCS 106, 012001 (2008).

23. Qiu, H. et al. Electrical characterization of back-gated bi-layer $\mathrm{MoS}_{2}$ field-effect transistors and the effect of ambient on their performances. Appl. Phys. Lett. 100, 123104 (2012).

24. Li, S.-L. et al. Thickness-dependent interfacial coulomb scattering in atomically thin field-effect transistors. Nano Lett. 13, 3546-3552 (2013).

25. Dolui, K., Rungger, I. \& Sanvito, S. Origin of the $n$-type and $p$-type conductivity of $\mathrm{MoS}_{2}$ monolayers on a $\mathrm{SiO}_{2}$ substrate. Phys. Rev. B 87, 165402 (2013).

26. Mott, N. F. Conduction in non-crystalline systems ix. the minimum metallic conductivity. Philos. Mag. 26, 1015-1026 (1972).

27. Bergmann, G. Weak localization in thin films: a time-of-flight experiment with conduction electrons. Phys. Rep 107, 1-58 (1984).

28. Akkermans, E. \& Montambaux, G. Mesoscopic Physics of Electrons and Photons (Cambridge University Press, 2007).

29. Brumme, T., Calandra, M. \& Mauri, F. First-principles theory of field-effect doping in transition-metal dichalcogenides: Structural properties, electronic structure, hall coefficient, and electrical conductivity. Phys. Rev. B 91, 155436 (2015).

30. Sodha, M. \& Eastman, P. Mobility of electrons in nondegenerate semiconductors considering electron-electron scattering. Z. Phys 150, 242-246 (1958).

31. Braga, D., Lezama, I. G., Berger, H. \& Morpurgo, A. F. Quantitative determination of the band gap of $\mathrm{WS}_{2}$ with ambipolar ionic liquid-gated transistors. Nano Lett. 12, 5218-5223 (2012).

32. Szabó, A., Rhyner, R. \& Luisier, M. Ab initio simulation of single- and few-layer $\mathrm{MoS}_{2}$ transistors: Effect of electron-phonon scattering. Phys. Rev. B 92, 035435 (2015). 
33. Bertolazzi, S., Brivio, J. \& Kis, A. Stretching and breaking of ultrathin $\mathrm{MoS}_{2}$. ACS Nano 5, 9703-9709 (2011).

34. Castellanos-Gomez, A. et al. Elastic properties of freely suspended $\mathrm{MoS}_{2}$ nanosheets. Adv. Mater. 24, 772-775 (2012).

35. Bao, W., Cai, X., Kim, D., Sridhara, K. \& Fuhrer, M. S. High mobility ambipolar $\mathrm{MoS}_{2}$ field-effect transistors: Substrate and dielectric effects. Appl. Phys. Lett. 102, 042104 (2013).

36. Chu, L. et al. Charge transport in ion-gated mono-, bi-, and trilayer $\mathrm{MoS}_{2}$ field effect transistors. Sci. Rep. 4, 1-4 (2014).

37. Halperin, B. I., Refael, G. \& Demler, E. Resistance in superconductors. Int. J. Mod. Phys. B 24, 4039-4080 (2010).

38. Benfatto, L., Castellani, C. \& Giamarchi, T. Broadening of the berezinskiikosterlitz-thouless superconducting transition by inhomogeneity and finite-size effects. Phys. Rev. B 80, 214506 (2009).

39. Jaeger, H. M., Haviland, D. B., Orr, B. G. \& Goldman, A. M. Onset of superconductivity in ultrathin granular metal films. Phys. Rev. B 40, 182-196 (1989).

40. Biscaras, J. et al. Multiple quantum criticality in a two-dimensional superconductor. Nat. Mater. 12, 542-548 (2013).

41. Jo, S., Costanzo, D., Berger, H. \& Morpurgo, A. F. Electrostatically induced superconductivity at the surface of $\mathrm{WS}_{2}$. Nano Lett. 15, 1197-1202 (2015).

42. Boukhicha, M., Calandra, M., Measson, M.-A., Lancry, O. \& Shukla, A. Anharmonic phonons in few-layer $\mathrm{MoS}_{2}$ : Raman spectroscopy of ultralow energy compression and shear modes. Phys. Rev. B 87, 195316 (2013).

\section{Acknowledgements}

This work was partly supported by French ANR project (Grant No. ANR-11-BS04-0019), by LABEX MATISSE through a post-doctoral grant and a scholarship from China

Scholarship Council (CSC, Grant No. 2011618135). We thank F. Gendron, J.-L. Cantin and J. Von Bardeleben for access to the electromagnet facility and M. Boukhicha for preliminary work. We acknowledge the Consortium des salles blanches dile de France, M. Rosticher and J. Palomo for access to clean room facilities and discussions with F. Mauri, M. Calandra and T. Brumme.

\section{Author contributions}

A.S. designed the project. Z.C. made the samples. Z.C. and A.P. fabricated the devices. J.B. performed the measurements and analysed the data. J.B. and A.S. wrote the paper.

\section{Additional information}

Supplementary Information accompanies this paper at http://www.nature.com/ naturecommunications

Competing financial interests: The authors declare no competing financial interests.

Reprints and permission information is available online at http://npg.nature.com/ reprintsandpermissions/

How to cite this article: Biscaras, J. et al. Onset of two-dimensional superconductivity in space charge doped few-layer molybdenum disulfide. Nat. Commun. 6:8826 doi: $10.1038 /$ ncomms9826 (2015).

This work is licensed under a Creative Commons Attribution 4.0 International License. The images or other third party material in this article are included in the article's Creative Commons license, unless indicated otherwise in the credit line; if the material is not included under the Creative Commons license, users will need to obtain permission from the license holder to reproduce the material. To view a copy of this license, visit http://creativecommons.org/licenses/by/4.0/ 